\title{
15. Über Beziehungen zwischen natürlicher und elektromagnetischer Rotationsdispersion $\left.{ }^{1}\right)$; von J. Disch.
}

Die vorliegenden Untersuchungen hatten vornehmlich den Zweck, an der Hand genauer Messungsergebnisse zu prüfen, ob das ron $G$. Wiedeman $n^{2}$ ) ausgesprochene Gesetz, wonach die magnetische Drehung der Polarisationsebene des Lichtes der bereits vorhandenen natürlichen Drehung proportional ist, strenge Gültigkeit besitzt. Zu diesem Zwecke bestimmte Verfasser für einige geeignete Substanzen sowohl die natürliche als auch die magnetische Drehung für verschiedene Farben des sichtbaren Spektrums.

Als Versuchsmaterial wurden nur klar durchsichtige aktive Substanzen gewählt, welche im Gebiete des sichtbaren Spektrums keine Absorption zeigen und optisch gut definiert sind, d. $h$. die nicht etwa eines inaktiven Lösungsmittels bedürfen. Um dieselben zu charakterisieren, bestimmte Verfasser deren Dichte und deren Brechungsexponent für dieselben Linien, für welche auch die Drehung gemessen wurde. Die Substanzen, welche zur Untersuchung gelangten, sind: deutsches, französisches und amerikanisches Terpentinöl, Zitronenöl, Paraffinöl, Valeriansäureäthyl, Diäthyltartrat und Quarz.

Als MeBmethode wurde diejenige gewählt, bei welcher man homogenes Licht in das Polaristrobometer fallen läßt, also inhomogenes Licht vorher reinigt. Diese Methode ist zwar umständlicher, gibt aber dafür zuverlässigere Resultate als die ältere von Broch ausgebildete Methode.

1) Auszug aus der gleichnamigen Inaugural-Dissertation von J. Dis ch, Freiburg i. B. 1903.

2) G. Wiedemann, Pogg. Ann. 82. p. 215. 1851; Die Lebre von der Elektrizität 3. p. 1054. 1895. 
Um sich homogenes Licht zu verschaffen, wurde das sehr intensive Licht der A r on s schen Quecksilberbogenlampe spektral zerlegt und die erforderliche Linie ausgeblendet; auf diese Weise wurden die Linien 578, 546, 491, 435 und $405 \mu \mu$ in großer Reinheit verwendet. Um die Natriumlinie zu erbalten, wurde eine Natriumflamme mit Kaliumbichromatlösung als Filter angewendet. Endlich lieB Verfasser den Induktionsfunken durch eine mit Wasserstoff gefüllte $G$ eisslersche Röhre schlagen, befreite das so erhaltene Licht durch Kaliumbichromatlösung und Rubinglas von seinen blauen und grünen Bestandteilen und erhielt auf diese Weise die Linie $656 \mu \mu$.

Als Polaristrobometer wurde ein Lippichscher Halbschattenapparat mit drei photometrischen Vergleichsfeldern verwendet, mit Hilfe dessen noch Tausendstelgrade abzulesen waren.

Die Flüssigkeiten wurden in eine Röbre von $60 \mathrm{~cm}$ Länge gegossen, die mit einer Kühlvorrichtung versehen war. Diese Röhre wurde in eine Spule gesteckt, welche im ganzen 1546 Windungen zählte und bei 12 Amp. Stromstärke ein Feld von ca. 600-700 C.G.S.- Einheiten lieferte. Der Quarz wurde in einer geeigneten Reguliervorrichtung zwischen die Pole eines durchbohrten Ruhmkorffschen Elektromagneten gebracht; wie sich aus der Drehung in Schwefelkohlenstoff berechnete, betrug bei $10 \mathrm{Amp}$. Stromstärke das Feld ca. 3800 C.G.S.Einbeiten.

Jede Flüssigkeit wurde vor ihrer Untersuchung mit Tierkohle gereinigt, jede Lichtquelle mit Hilfe eines Handspektroskopes auf ihre Homogenität hin geprüft; desgleichen wurde strenge auf Erzielung konstanter Temperatur geachtet. Die natürlichen und magnetischen Drehungen ergaben sich aus den Einstellungen bei leerem Apparat, mit eingeschobener aktiver Substanz ohne Magnetfeld und im Felde. Je nach der Intensität der Lichtquelle betrug die GröBe des Halbschattens $3-30^{\circ}$ und die Zahl der Einstellungen 5 bis 40 .

Seine Messungsergebnisse hat Verfasser sowohl in Tabellen zusammengestellt als auch durch Kurven graphisch veranschaulicht. Der Kürze halber seien als Beispiele hier nur Valeriansäureäthyl und Quarz angeführt. Unter $n$ bez. $m$ sind die natürlichen bez. magnetischen Drehungen für die Wellenlängen $\lambda$ 
angeführt. $n / n_{e 58}$ und $m / m_{658}$ bezeichnen die Drehungsverhältnisse, wenn die Drehung für $656 \mu \mu$ gleich 1 gesetzt wird. Bei der graphischen Darstellung sind als Abszissen die Wellenlängen, als Ordinaten die Drehungsverhältnisse aufgetragen; es gibt dann die Divergenz je zweier entsprechender Kurven direkt ein $\mathrm{MaB}$ für die Abweichung vom Wiedemannschen Gesetz.

\begin{tabular}{cccccc}
\multicolumn{5}{c}{ Valeriansäureäthyl. } \\
$\lambda$ & $n$ & $m$ & $n / m$ & $n / n_{\text {658 }}$ & $m / m_{\text {85B }}$ \\
656 & $\mathbf{1 3 , 3 6 7}$ & 3,825 & 3,495 & 1,000 & 1,000 \\
589 & 16,814 & 4,462 & 3,768 & 1,258 & 1,167 \\
546 & 20,045 & 5,278 & 3,798 & 1,500 & 1,380 \\
492 & 25,717 & 6,677 & 3,852 & 1,924 & 1,746 \\
436 & 34,393 & 8,671 & 3,966 & 2,573 & 2,267 \\
\multicolumn{5}{c}{ Quarz. } \\
656 & 17,313 & 2,933 & 5,903 & 1,000 & 1,000 \\
589 & 21,720 & 3,668 & 5,921 & 1,254 & 1,251 \\
578 & 22,632 & 3,817 & 5,929 & 1,307 & 1,301 \\
546 & 25,532 & 4,295 & 5,945 & 1,475 & 1,464 \\
492 & 31,967 & 5,340 & 5,986 & 1,846 & 1,821 \\
436 & 41,548 & 6,792 & 6,117 & 2,400 & 2,316 \\
405 & 48,930 & 7,838 & 6,243 & 2,826 & 2,672
\end{tabular}

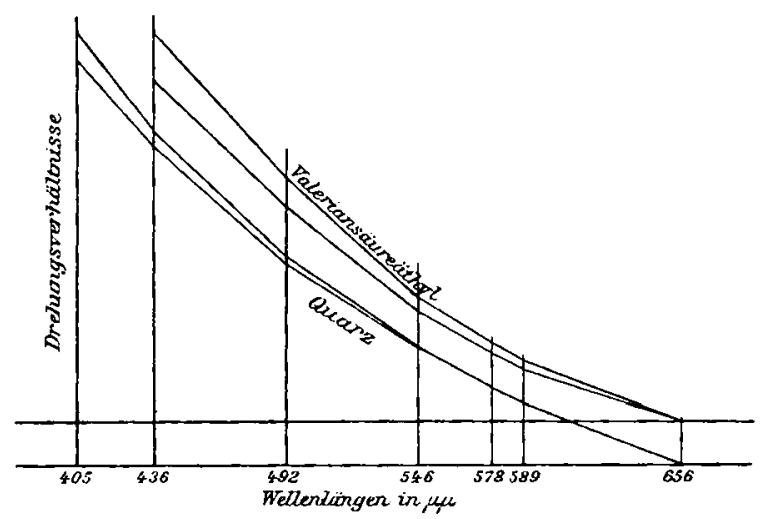

Sowohl die Tabellen als auch die graphische Darstellung ergeben, daB das Wiedemannsche Gesetz mit groBer Annäherung gilt; der Quotient $n / m$ ist so gut wie konstant, die Verhältnisse für die natürlichen und magnetischen Drehungen 
sind einander nahezu gleich und dementsprechend fallen je zwei Kurven fast zusammen. Ebenso wie Valeriansäureäthyl verhalten sich deutsches und französisches Terpentinöl, Paraffinöl, Zitronenöl. Die Abweichungen, welche zweifelsohne regelmäBiger Natur sind, wie auch die Berücksichtigung der Beobachtungsfehler ergibt $\left.{ }^{1}\right)$, sind am geringsten für Quarz, ja dieselben verschwinden ganz, wenn man die Kurve der natürlichen Drehungen vergleicht mit der Mittelkurve aus den Messungen über magnetische Drehung von Borel und von mir. ${ }^{2}$ ) Die Tatsache nun, daß der Quarz bezüglich seiner Aktivität sehr einheitlich ist, d. h. daB rechtsdrehender Quarz keine Spur von linksdrehendem enthält und umgekehrt, führte den Verfasser zu der Ansicht, daB überhaupt alle reinen aktiven Substanzen dasselbe Verhalten wie Quarz aufweisen und daB die jeweils zu beobachtenden Abweichungen von aktiven Beimengungen herrühren. $\mathrm{Ob}$ diese letzteren für die drehenden organischen Flüssigkeiten im Racemat oder in aktiven Molekülaggregaten zu suchen seien, ist schwer zu entscheiden. Verfasser schlieBt sich der letzteren Ansicht an; danach müBten bei einer Substanz, die nur drehende Moleküle enthält, natürliche und magnetische Rotationsdispersion genau zusammen. fallen, durch die aktiven Molekülkomplexe würde die natürliche Drehungsdispersion modifiziert und dadurch die Abweichungen entstehen, welche je nach der Anzahl der Aggregate gering oder bedeutend sein können, wie dies letztere bei $\mathrm{Di}$ äthyltartrat und amerikanischem Terpentinöl der Fall ist.

Was die Theorie der Erscheinung anbelangt, so hat Verfasser die drei Gleichungen von Boltzmann ${ }^{3}$, Carvallo ${ }^{4}$ ) und Drude ${ }^{5}$ ), welche die natürliche Drehung als Funktion der Wellenlänge darstellen, untersucht und gefunden, daB dieselben auch die magnetische Drehung in befriedigender Weise darstellen, d. h. die Unterschiede zwischen den beobachteten und den nach den betreffenden Gleichungen berechneten magnetischen Drehungen liegen innerhalb der Grenzen der Versuchsfehler.

1) Vgl. ausführliches hierüber in der Dissertation, Abschnitt $X$.

2) l. c.

3) 4) 5) Vgl. hierüber in Abschnitt XI der Dissertation. 
Natürliche und elektromagnetische Rotationsdispersion. 1157

Verfasser kommt also durch seine Untersuchungen zu folgenden Resultaten:

1. Die von G. Wiedemann gefundene GesetzmäBigkeit wird nur von solchen Substanzen streng befolgt, welche hinsichtlich ihrer Aktivität einheitlich sind.

2. Je strenger eine Substanz jenem Gesetze gehorcht, desto reiner ist sie bezüglich ihrer optischen Aktivität.

3. Die für die natürliche Rotationsdispersion aufgestellten Gleichungen können auch zur Darstellung der magnetischen Drehungsdispersion dienen.

Freiburg i. B., Physik. Institut, im Juli 1903.

(Eingegangen 21. August 1903.) 